\title{
Alk5 inhibition increases delivery of macromolecular and protein-bound contrast agents to tumors
}

Heike E. Daldrup-Link, ${ }^{1}$ Suchismita Mohanty, ${ }^{1}$ Celina Ansari, ${ }^{1}$ Olga Lenkov, ${ }^{1}$ Aubie Shaw, ${ }^{2}$ Ken Ito, ${ }^{1}$ Su Hyun Hong, ${ }^{1}$ Matthias Hoffmann, ${ }^{3}$ Laura Pisani, ${ }^{1}$ Nancy Boudreau, ${ }^{4}$ Sanjiv Sam Gambhir, ${ }^{1,5,6}$ and Lisa M. Coussens ${ }^{2}$

'Department of Radiology, Molecular Imaging Program at Stanford (MIPS), Stanford University, Stanford, California, USA. 2Department of Cell, Developmental and Cancer Biology, Knight Cancer Institute, Oregon Health and Science University, Portland, Oregon, USA. ${ }^{3}$ Department of Dermatology, Venereology and Allergology, Goethe University, Frankfurt, Germany. ${ }^{4}$ Department of Surgery, UCSF, San Francisco, California, USA. ${ }^{5}$ Department of Bioengineering and ${ }^{6}$ Department of Materials Science and Engineering, Stanford University, Stanford, California, USA.

Limited transendothelial permeability across tumor microvessels represents a significant bottleneck in the development of tumor-specific diagnostic agents and theranostic drugs. Here, we show an approach to increase transendothelial permeability of macromolecular and nanoparticle-based contrast agents via inhibition of the type I TGF- $\beta$ receptor, activin-like kinase 5 (Alk5), in tumors. Alk 5 inhibition significantly increased tumor contrast agent delivery and enhancement on imaging studies, while healthy organs remained relatively unaffected. Imaging data correlated with significantly decreased tumor interstitial fluid pressure, while tumor vascular density remained unchanged. This immediately clinically translatable concept involving Alk5 inhibitor pretreatment prior to an imaging study could be leveraged for improved tumor delivery of macromolecular and nanoparticle-based imaging probes and, thereby, facilitate development of more sensitive imaging tests for cancer diagnosis, enhanced tumor characterization, and personalized, image-guided therapies.

Authorship note: H.E. Daldrup-Link and S. Mohanty contributed equally to this work.

Conflict to interest: The authors have declared that no conflict of interest exists.

Submitted: November 18, 2015 Accepted: March 29, 2016 Published: May 5, 2016

Reference information: JCI Insight. 2016;1(6):e85608. doi:10.1172/jici.insight.85608.

\section{Introduction}

Improved understanding of cellular, molecular, and genomic characteristics of malignant tumors has spurred development of a wide range of tumor-targeted imaging probes designed to provide more sensitive and specific information about the underlying tumor, as compared with conventional, nonspecific contrast agents (1-9). Previous studies have reported that nanoparticle-based imaging probes, dendrimers, micelles, and liposomal diagnostic and theranostic agents, among many others, significantly improve tumor detection, characterization, and therapy stratification and improve selective delivery of anticancer drugs (1-9). However, one major limitation in successful clinical translation of these imaging agents is their large size, which limits their delivery to tumor parenchyma or other target cells in tumor microenvironments $(1,2,7)$; thus, only a fraction of their maximal potential is being realized in the clinic. The two major barriers for efficient delivery of macromolecular drugs to tumor parenchyma include the altered permeability of tumor microvessels and high interstitial fluid pressure (IFP) in solid tumors (10-14). Thus, strategies must be developed to overcome these barriers in order to maximize the clinical impact of macromolecular imaging agents.

Agents that promote vascular leakage are effective in increasing tissue accumulation of macromolecular drugs by virtue of their ability to overcome high IFP and allow convection-driven uptake into tissues $(10,11,15-17)$. TGF- $\beta 1$ restricts both homeostatic and tumoral vascular leakage, while inhibition of the type I TGF- $\beta$ receptor, activin-like kinase 5 (Alk5), expressed by vascular cells leads to enhanced vascular leakage in mammary and cutaneous carcinomas (18). Since clinically applicable Alk5 inhibitors are readily available and could be applied prior to administration of diagnostic or theranostic drugs (19), we evaluated if Alk5 inhibition could improve the tumor delivery and enhancement of clinically applicable macromolecular contrast agents in vivo. By exploiting an endogenous pathway that regulates vascular leakage and remains functional during cancer development, this approach significantly improved tumor delivery of FDA-approved contrast agents and nanocarriers, without significantly altering hemodynamics of normal 
Table 1. Experimental groups

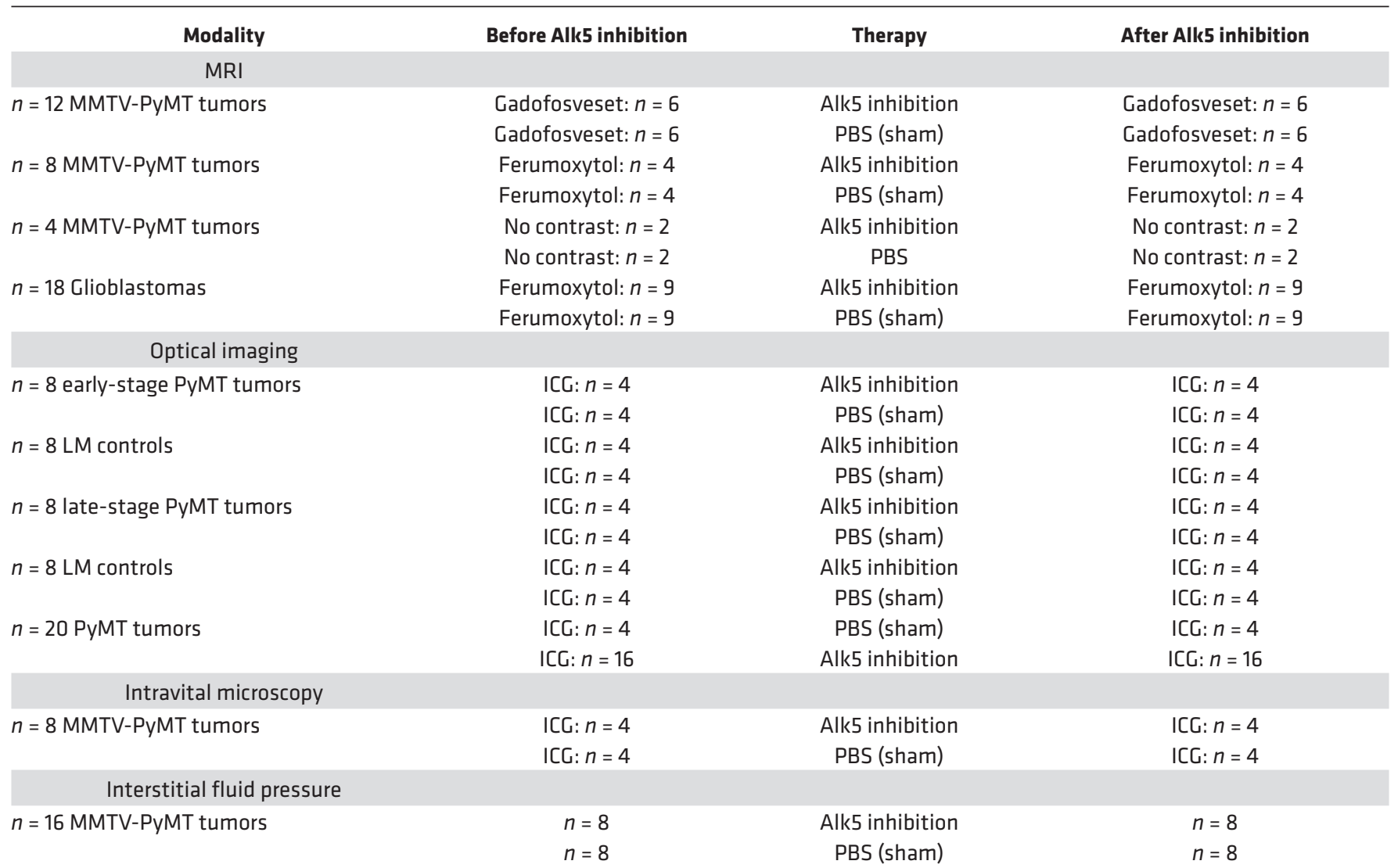

MMTV-PyMT, mouse mammary tumor virus-driven expression of the polyoma middle T oncogene; ICG, indocyanine green; LM, negative littermate.

organs. Based on these findings, we predict that this easily applicable intervention, prior to an imaging study, will enhance sensitivity and specificity of imaging tests for cancer diagnosis, improve tumor characterization, and improve therapeutic response to personalized and image-guided therapies.

\section{Results}

We examined 100 mice bearing transgenic mouse mammary tumor virus-driven expression of the polyoma middle T oncogene (MMTV-PyMT) adenocarcinomas (20) or non-tumor-bearing control littermates before and after i.p. administration of the Alk5 kinase inhibitor [3-(pyridin-2-yl)-4-(4-quinonyl)]-1H-pyrazole. For some experiments (see Methods), explants derived from MMTV-PyMT tumors were investigated in female syngeneic FVB/n mice $(21,22)$. To confirm findings in a second tumor model, we examined $18 \mathrm{NOD} / \mathrm{SCID} \times \mathrm{RAG} \gamma / \delta$ double-knockout mice with an orthotopically implanted D270 glioblastoma cell line derived from human brain tumors. Both MMTV-PyMT tumors (23) and glioblastomas (24) have elevated TGF- $\beta$ signaling activity.

Alk5 inhibition increases tumor enhancement on magnetic resonance images. We first evaluated the tumor delivery of gadofosveset trisodium $\left(\mathrm{C}_{33} \mathrm{H}_{40} \mathrm{GdN}_{3} \mathrm{Na}_{3} \mathrm{O}_{15} \mathrm{P}\right.$; Ablavar, dose $\left.0.05 \mathrm{mmol} \mathrm{Gd} / \mathrm{kg}\right)$, an FDA-approved small molecular magnetic resonance (MR) contrast agent that binds $>90 \%$ to plasma albumin and thereby forms macromolecules of approximately 80 to $90 \mathrm{kDa}$ in vivo (25). MMTV-PyMT mice injected with gadofosveset trisodium demonstrated a significant positive (hyperintense) tumor enhancement on T1-weighted MR images (Table 1 and Figure 1). This tumor enhancement, quantified as $\Delta \mathrm{R} 1$ data, increased by 3-fold following Alk5 inhibitor treatment as compared with untreated controls $(P<0.05$; Supplemental Figure 2; supplemental material available online with this article; doi:10.1172/jci.insight.85608DS1). There was no significant effect of Alk5 inhibition on gadofosveset enhancement of normal non-tumor-bearing organs (Figure $1 ; P>0.05$ ). 


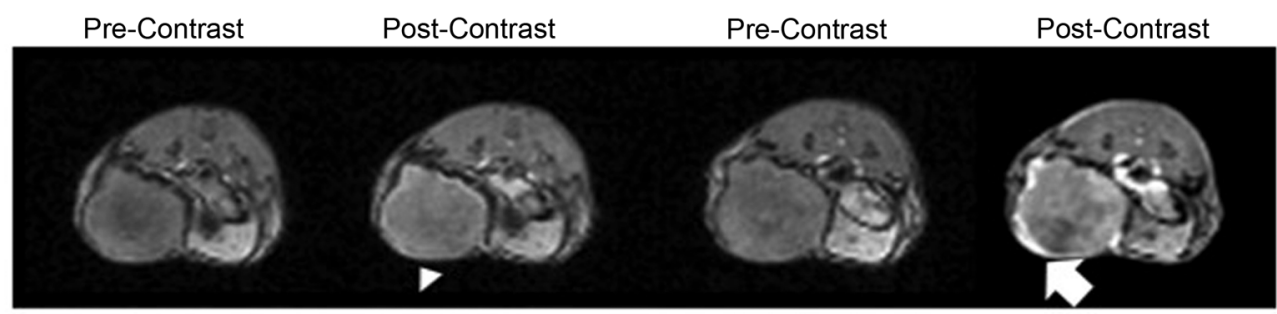

B

Gadofosveset Trisodium Enhancement
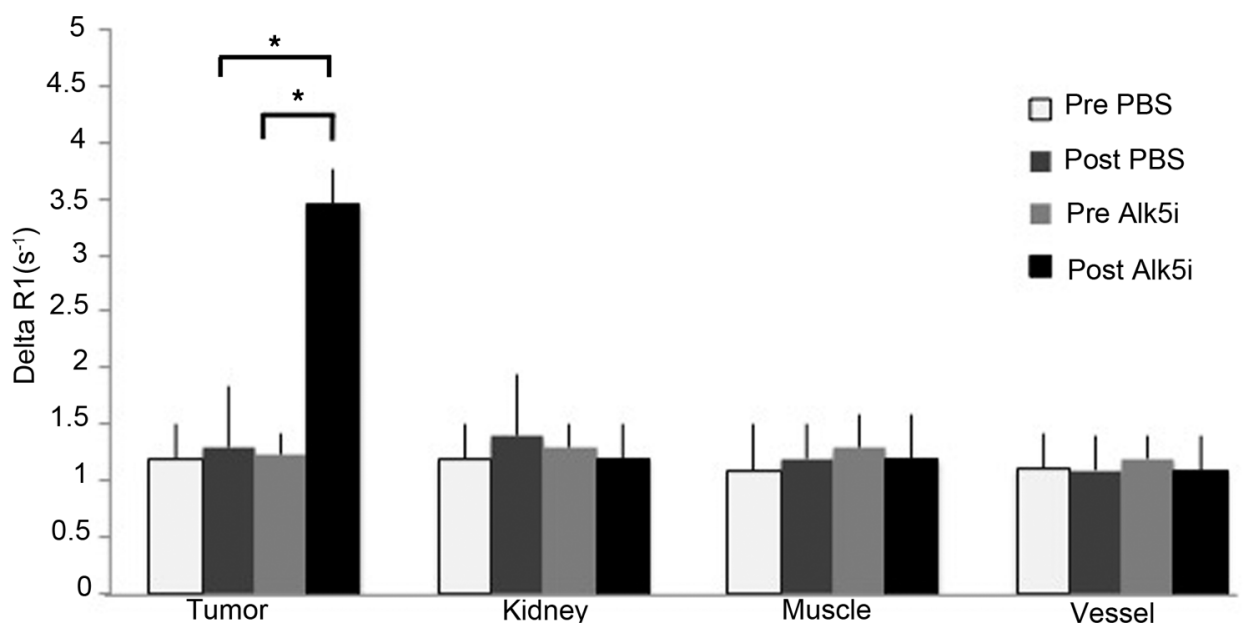

Figure 1. Alk5 inhibition leads to increased gadofosveset trisodium accumulation and enhancement of MMTV-PyMT tumors on MR images. (A) Representative axial T1-weighted MR images before and after i.v. injection of gadofosveset trisodium show stronger positive (bright) tumor enhancement after Alk5 inhibition (arrow) as compared with untreated tumors (arrowhead). (B) Corresponding quantitative changes in tumor R1 relaxation rates, measured as $\mathrm{R} 1_{\text {precontrast }}-\mathrm{R} 1_{\text {postcontrast }}$ and displayed as means for 6 animals in each group $\pm \mathrm{SD}$, confirm significantly increased tumor enhancement after Alk5 inhibition (Alk5i). ANOVA was used to compare the differences between various groups. ${ }^{*} P<$ 0.05 , between MR enhancement of Alk5 inhibitor-treated tumors compared with controls. Results are representative of 3 independent experiments. Alk5, activin-like kinase 5; MMTV-PyMT, mouse mammary tumor virus-driven expression of the polyoma middle T oncogene; MR, magnetic resonance.

We next evaluated the tumor delivery of a classical FDA-approved iron oxide nanoparticle compound, ferumoxytol (Feraheme; dose $0.5 \mathrm{mmol} \mathrm{Fe} / \mathrm{kg}$ ) (26). Mammary tumors in MMTV-PyMT mice injected with ferumoxytol showed negative (hypointense) T2 enhancement on T2-weighted MR images (Figure 2). Alk5 inhibition improved mean tumor enhancement by 2 -fold compared with untreated controls $(P<0.05)$. Increasing tumor size increased Alk5 inhibitor-mediated effects on both gadofosveset and ferumoxytol delivery to tumors (Supplemental Figure 1). Importantly, while Alk5 inhibition enhanced delivery of gadofosveset to the vascular-rich tumor perimeter, the delivery of ferumoxytol was enhanced to both the tumor periphery and center. Mammary tumors from control mice treated with PBS showed no significant changes in tumor enhancement (Figure 2; $P>0.05$ ), and, similarly, mice treated with Alk5 inhibitor but not injected with contrast agent also showed no change in MR signal. Normal non-tumor-bearing organs (e.g., liver, spleen, kidney, paraspinal muscle) did not show significant changes in MRI enhancement after Alk5 inhibitor treatment (Figure 2; $P>0.05$ ).

Histopathologic correlations confirmed increased interstitial accumulation of ferumoxytol nanoparticles in Alk5 inhibitor-treated MMTV-PyMT tumors as compared with untreated controls, as indicated by increased presence of iron (Figure 3). Conversely, homeostatic organs did not show significant changes in ferumoxytol accumulation after Alk5 inhibition (data not shown). Tumor tissue from Alk5 inhibitor-treated mice exhibited reduced Alk5 and phospho-Smad 2 throughout tumors, indicating reduced TGF- $\beta$ signaling capacity without any altered phenotype of CD31-positive vessels within mammary tumors (Figure 3 and Supplemental Figures 3 and 4). 
A

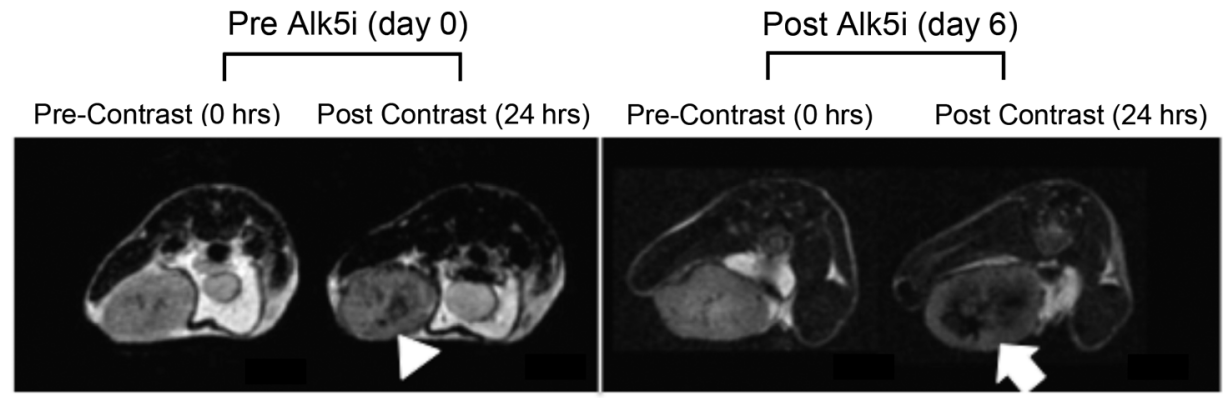

B

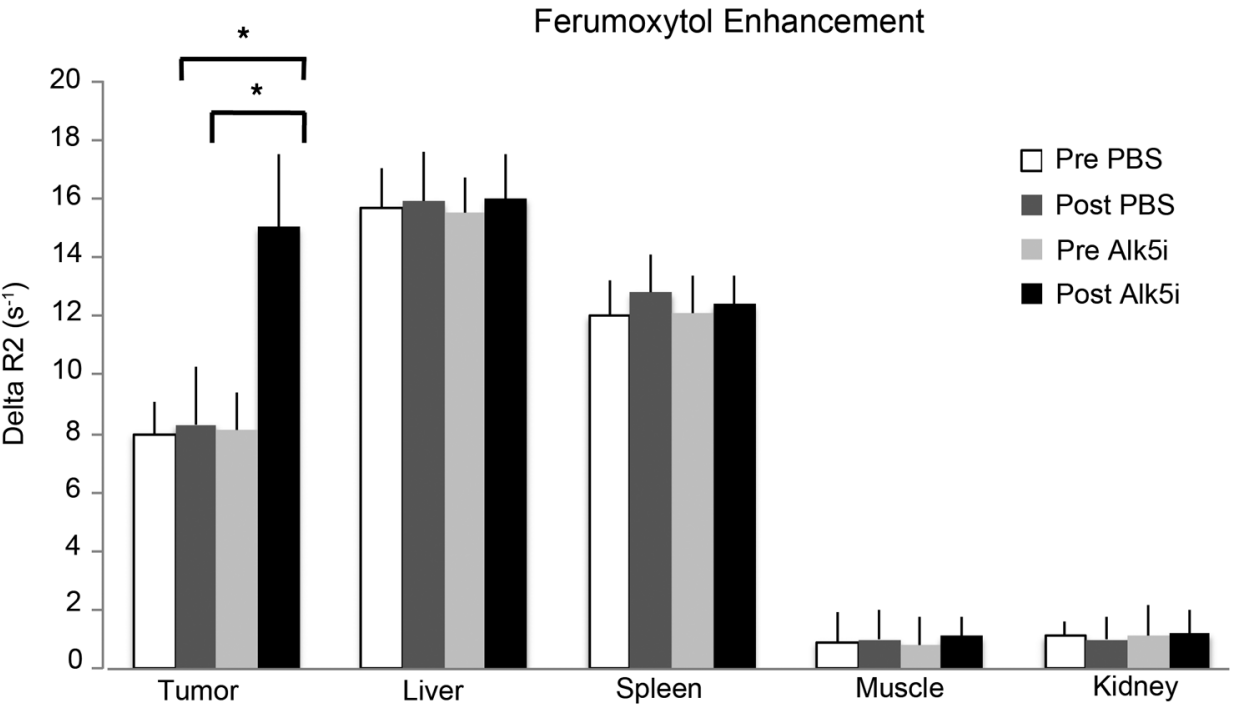

Figure 2. Alk5 inhibition leads to increased iron oxide nanoparticle accumulation and enhancement of MMTV-

PyMT tumors on MR images. (A) Representative axial T2-weighted MR images before and after i.v. injection of ferumoxytol nanoparticles show stronger negative (dark) tumor enhancement after Alk5 inhibition (arrow) compared with untreated tumors (arrowhead). (B) Corresponding quantitative changes in tumor R2 relaxation rates after ferumoxytol injection, measured as $\mathrm{R} 2_{\text {precontrast }}-\mathrm{R} 2_{\text {postcontrast }}$ and displayed as means for 4 animals in each group \pm SD. ANOVA was used to compare the differences between various groups. ${ }^{*} P<0.05$, between MR enhancement of Alk5 inhibitor-treated tumors compared with untreated controls. Results are representative of 3 independent experiments. Alk5, activin-like kinase 5; Alk5i, Alk5 inhibition; MMTV-PyMT, mouse mammary tumor virus-driven expression of the polyoma middle T oncogene; MR, magnetic resonance.

Alk5 inhibition increases tumor enhancement on optical images. Next, we investigated the effects of Alk5 inhibition on tumor enhancement on optical images and effects of Alk5 inhibition on contrast agent delivery to early-stage cancers (day 50) and late-stage cancers (day 75) in transgenic MMTV-PyMT mice. i.v. injection of the FDA-approved fluorescent marker indocyanine green (ICG), which binds $80 \%$ to $90 \%$ to plasma proteins, caused a marked tumor enhancement on optical scans (Figure 4A). The ICG tumor enhancement increased significantly following Alk5 inhibition (Figure $4, P<0.05$ ) and was significantly higher as compared with either tumors from untreated mice or normal mammary fat pad $(P<0.05$; Figure 4B). Optical images before the first and second ICG injection showed similar tumor signal, confirming that no contrast enhancement had remained from the first pre-Alk5 inhibition imaging session. ICG-enhanced optical scans revealed a relatively stronger effect of the Alk5 inhibitor on late-stage cancers, with an average 3.5-fold gain in tumor enhancement, compared with early-stage cancers, with an average 2-fold gain (Figure 4B). We further performed a dose-response study, demonstrating that increasing doses of Alk5 inhibitor $(0.5,1,2$, and $4 \mathrm{mg} / \mathrm{kg}$ mouse body weight) consistently enhanced ICG signals compared with vehicle-treated animals (Figure 5). Importantly Alk5 inhibition also reduced IFP in tumors, as observed in Figure 4C $(P<0.05)$. Intravital microscopy studies further confirmed significantly increased ICG delivery to Alk5 inhibitor-treated tumors compared with untreated controls $(P<0.05$; Figure 6$)$, without change in tumor vascular density (Figure 6 and Supplemental Figure 5). 

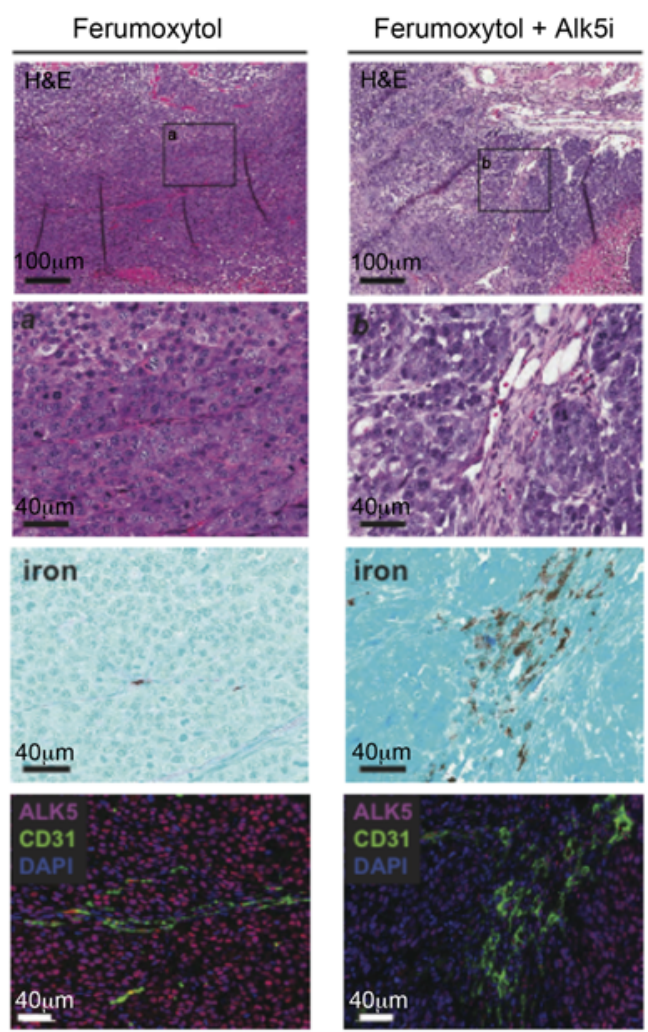

Figure 3. Representative histology of MMTV-PyMT mammary tumors indicates increased ferumoxytol accumulation after Alk5 inhibition. (Top) H\&E staining of formalin-fixed paraffin-embedded tissue sections of MMTV-PyMT tumors (original magnification, $\times 10$; scale bar: $100 \mu \mathrm{m}$ ), with boxed regions shown below at higher magnification (original magnification, $x 40$; scale bar: $400 \mu \mathrm{m}$ ) in images below. Prussian blue staining was used to reveal ferumoxytol/iron in adjacent tissue sections. (Bottom) Immunofluorescent staining for CD31 (green) and Alk5 (red) was conducted in adjacent tissue sections from the tissue block. Results are representative of 3 independent experiments. Scale bar: $40 \mu \mathrm{m}$. Alk5, activin-like kinase 5; Alk5i, Alk5 inhibition; MMTV-PyMT, mouse mammary tumor virus-driven expression of the polyoma middle T oncogene.

Confirmation in a second tumor model with mice bearing glioblastomas. To confirm the finding that Alk5 inhibition increased tumor delivery of imaging agents, we next examined the delivery of ferumoxytol nanoparticles to human glioblastomas implanted orthotopically in NOD/SCID $\times$ RAG $\gamma / \delta$ double-knockout mice before and after Alk5 inhibition. Consistent with our results in MMTV-PyMT adenocarcinomas, we observed that Alk5 inhibitor treatment prior to contrast agent administration markedly enhanced ferumoxytol delivery into brain tumors, as demonstrated by marked tumor enhancement on T1- and T2-weighted MR images (Figure 7A). Mice treated with PBS showed only minor tumor enhancement at 24 hours after contrast agent delivery (Figure 7A). Dynamic ferumoxytol-enhanced MRI scans demonstrated markedly increased tumor enhancement of Alk5-treated tumors compared with untreated controls (Figure 7, B and C). Corresponding quantitative $\Delta \mathrm{R} 1$ and $\Delta \mathrm{R} 2$ data at 24 hours after contrast agent delivery showed more than a 2 -fold increase in tumor enhancement when compared with PBS-treated groups (Figure 7, D and E; $P<0.05)$. Prussian blue staining of the tumor tissue confirmed a selective ferumoxytol enrichment in brain tumors of animals treated with Alk5 inhibitor (Figure 7F and Supplemental Figure 6), with minor accumulation in untreated tumors (Figure 7F) and no accumulation in normal tissues (data not shown).

\section{Discussion}

Our study demonstrates that therapeutic inhibition of a vascular stabilizing pathway regulated by the TGF- $\beta 1$ receptor, Alk5, substantially improves delivery of macromolecular and protein-bound contrast agents to malignant tumors, without altering hemodynamics of homeostatic tissues/organs. This approach of inhibiting TGF- $\beta 1$ could be immediately utilized clinically to improve the delivery of tumor-targeted macromolecular and nanoparticle-, liposome-, micelle-, and antibody-based diagnostic and theranostic drugs for better disease diagnosis $(10,11,14)$.

Tumor accumulation of i.v. administered diagnostic and theranostic drugs depends on several variables, including blood flow, transendothelial solute/drug permeability, IFP, and interstitial volume, (10-16, 27). Several investigators reported that reduced permeability of tumor microvessels limits delivery of macromolecular drugs, and the high IFP in solid tumors reduces cytotoxic drug extravasation from tumor vessels into tumor parenchyma (10-14, 27). A recent study comparing 3 different, commonly used murine tumor models and 2 nanoparticle-based diagnostic drugs revealed significant extravasation of nanoparti- 

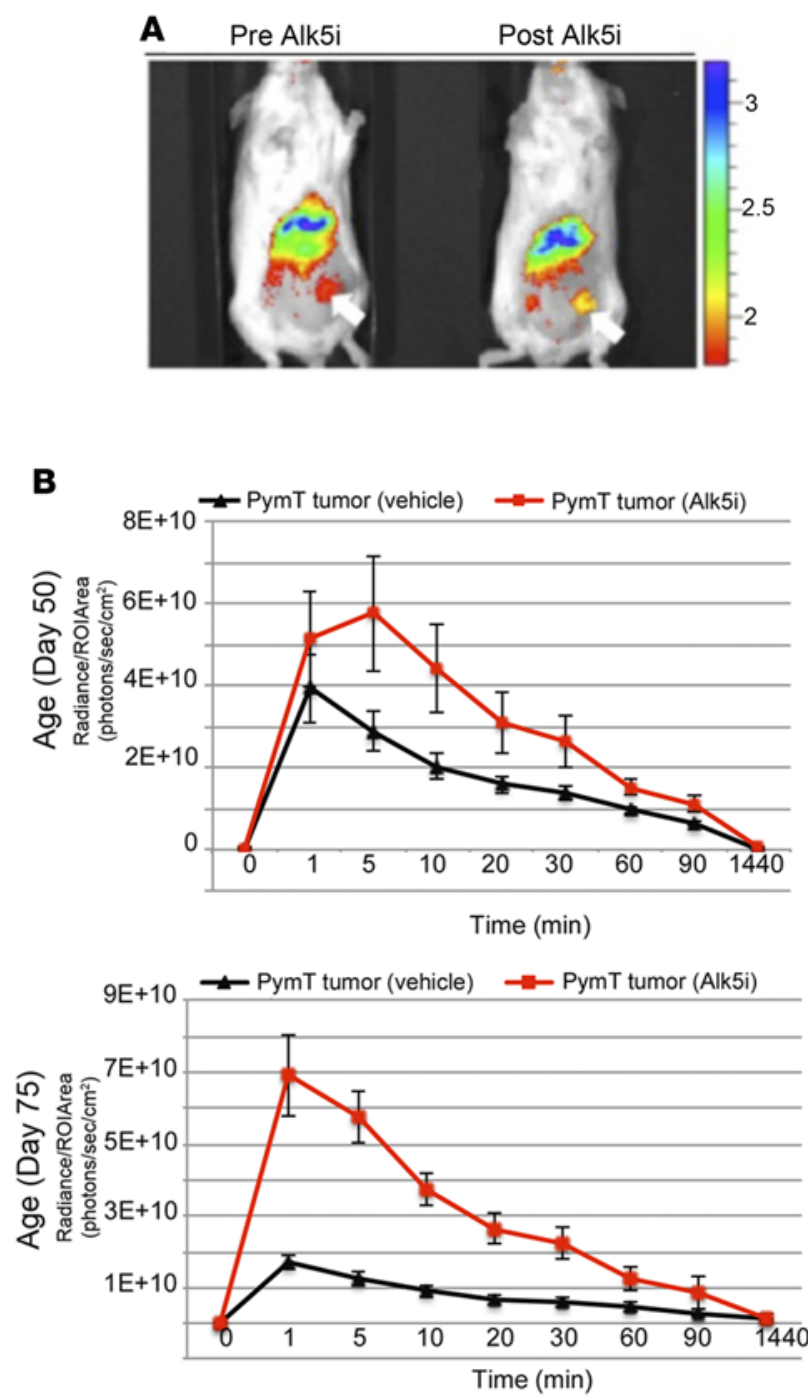

C Interstitial Fluid Pressure $(\mathrm{mm} \mathrm{Hg})$
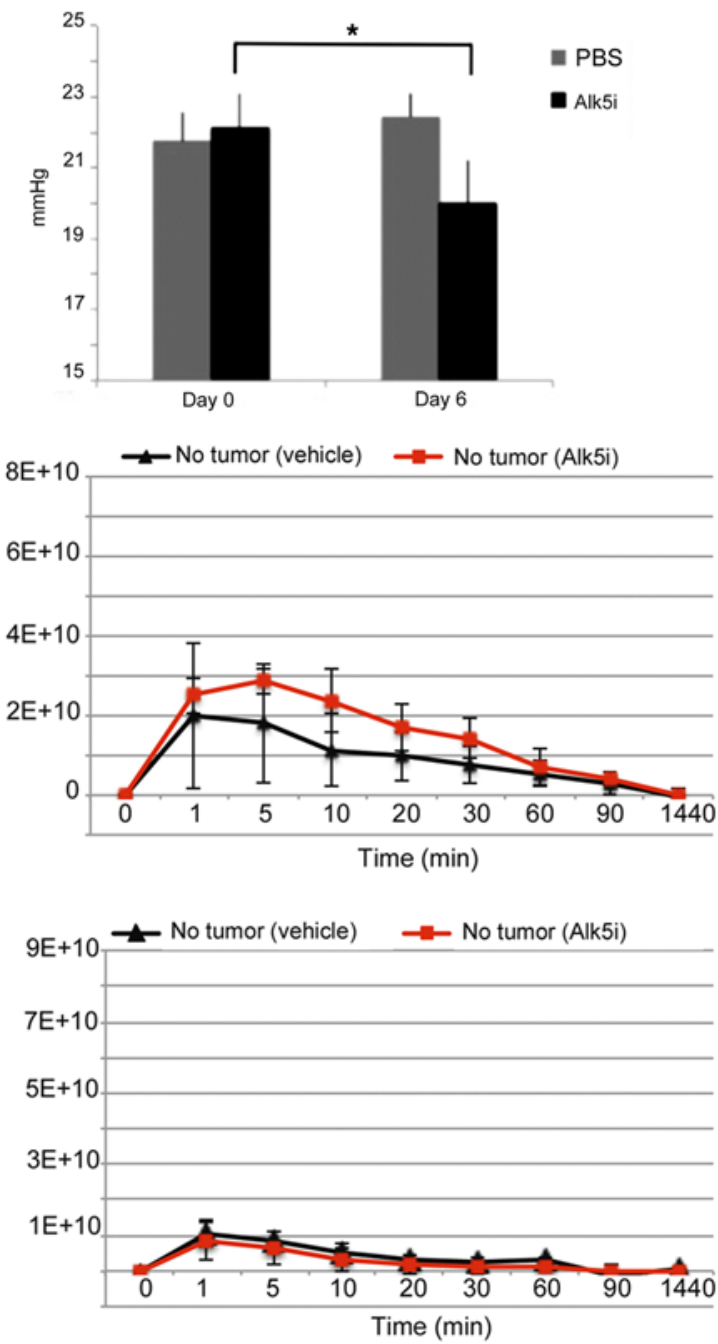

Figure 4. Alk5 inhibition leads to increased indocyanine green accumulation and enhancement of MMTV-PyMT tumors on optical images. (A) Representative optical scans demonstrate higher fluorescence in an Alk5 inhibitor-treated tumor as compared with an untreated age-matched tumor (arrows). Liver fluorescence is not significantly different. (B) Quantitative fluorescence signal of early-stage (day 50) and late-stage (day 75) MMTV-PyMT tumors before and after treatment with Alk5 inhibitor. The breast fat pad of non-tumor-bearing mice served as controls. Alk5 inhibition led to improved indocyanine green (ICG) tumor enhancement of both early- and late-stage cancers; the effect is relatively stronger in late-stage cancers. Data are displayed as mean ( \pm SD) tumor fluorescence of 4 animals in each group. (C) Tumor interstitial fluid pressure (IFP) measurements revealed significantly reduced IFP in Alk5 inhibitor-treated tumors compared with untreated controls. Data are displayed as mean tumor fluorescence of 8 animals in each group with SD. ANOVA was used to compare the differences between various groups. ${ }^{*} P<0.05$. Results are representative of 3 independent experiments. Alk5, activinlike kinase 5; Alk5i, Alk5 inhibition; MMTV-PyMT, mouse mammary tumor virus-driven expression of the polyoma middle T oncogene.

cles only for 2 of 6 conditions (28), thereby indicating that strategies to overcome or exploit tumor barriers are critically needed. Although antibodies against VEGF or its receptor can transiently increase tumor perfusion and penetration of chemotherapeutic agents, this strategy is largely limited to immature vessels in early-stage tumors lacking pericyte or smooth muscle cell coverage (29-31), whereas mature vessels in later-stage tumors are often refractory to VEGF blockade (32). Moreover, VEGF blockade only increases tumor delivery of intermediate size (12-nm), but not larger (125-nm), nanoparticles (33). Since most diagnostic contrast agent and theranostic nanoparticles range from 30 to $200 \mathrm{~nm}$ (2, 3, 34-36), additional approaches are necessary to effectively increase tumor delivery of these probes.

Our data showed that Alk5 inhibition increased tumor contrast agent enhancement by increasing transendothelial delivery of contrast agents and decreasing IFP. Bhattacharya et al. suggested that small molecule therapeutic inhibitors of TGF- $\beta$, including Alk 5 inhibitors, are less expensive when compared with conventional recombinant therapeutic agent such as antibodies and could avoid complications asso- 
A Alk5i doses (mg/kg body weight)
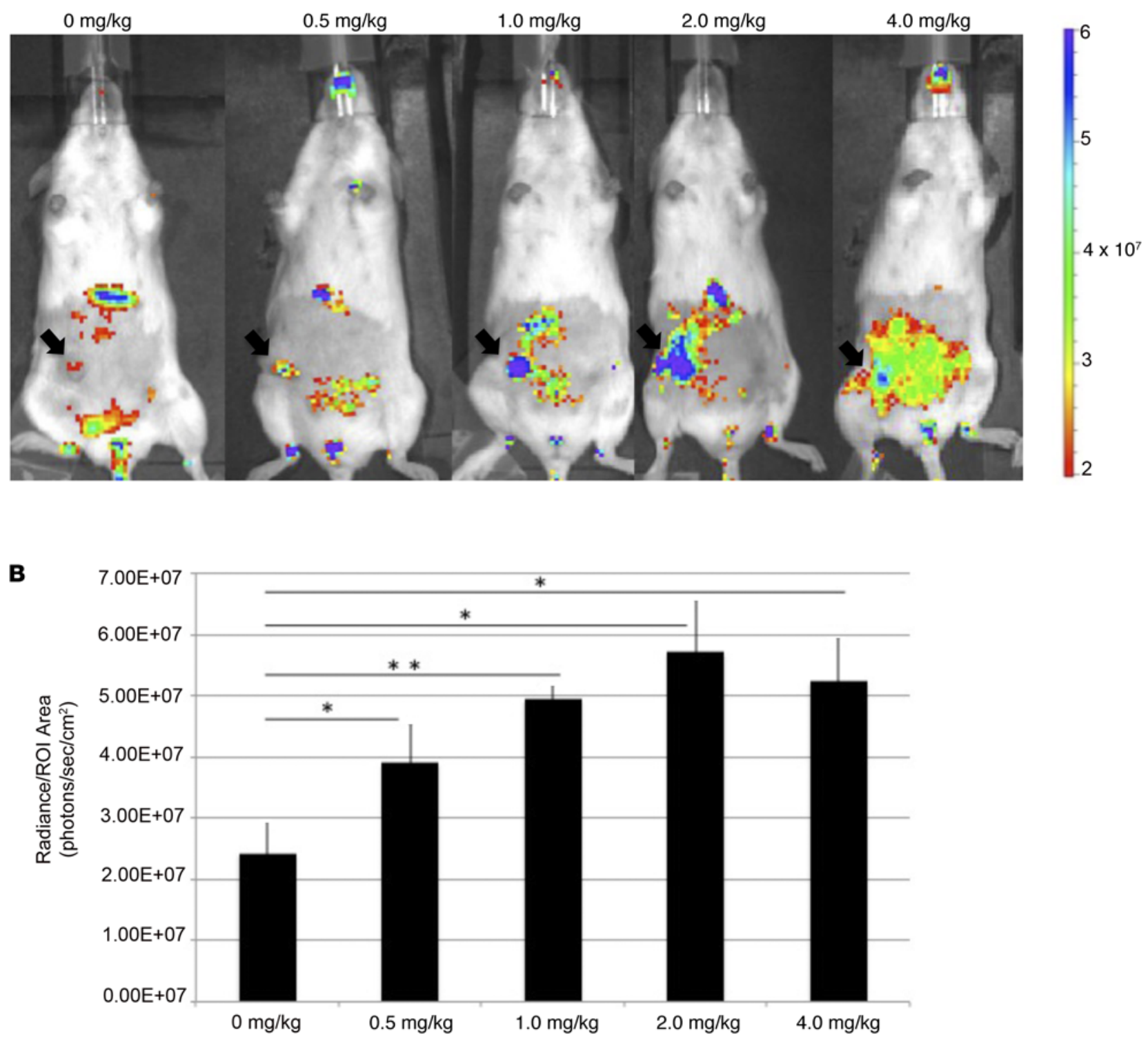

Figure 5. Increased accumulation of indocyanine green in MMTV-PyMT tumors following Alk5 inhibition at different doses. (A) Representative optical scans demonstrate higher fluorescence at increasing doses of Alk5 inhibitor treatment as compared with vehicle treated age-matched tumor (arrows). Liver fluorescence is not significantly different. (B) Quantitative fluorescence signal of vehicle- and Alk5 inhibitor-treated MMTV-PyMT tumors. Data are displayed as mean tumor fluorescence of 4 animals in each group $( \pm$ SD). ANOVA was used to compare the differences between various groups. ${ }^{*} P<0.05,{ }^{* *} P<0.005$. Alk5, activin-like kinase 5; Alk5i, Alk5 inhibition; MMTV-PyMT, mouse mammary tumor virus-driven expression of the polyoma middle $T$ oncogene.

ciated with recombinant therapeutic agents (37). The TGF- $\beta 1$ pathway, via its receptor Alk5, is known to increase collagen synthesis in tumors, and blocking this pathway increases vessel perfusion and decreases resistance of the interstitial matrix (18). This reduces IFP of tumors, leading to the improved delivery of both low and high molecular weight agents. In agreement with our earlier findings (18), studies reveal that TGF- $\beta 1$ blockade decreased IFP in murine models of thyroid cancer (38) and increased tumor microvascular permeability to various macromolecular drugs in murine pancreatic (39), squamous, and mammary cancers $(18,40)$. Mechanisms of how TGF- $\beta$ regulates vessel permeability have been described previously. TGF- $\beta$ differentially affects endothelial and vascular smooth muscle cells in a concentration-, substrate-, and tissue-specific context $(41,42)$. For example, in bleomycin-induced pulmonary edema, TGF- $\beta$ promotes vascular leakage by acting directly on pulmonary endothelial cells in concert with integrin $\alpha 5 \beta 1$ ( 43 , 
A

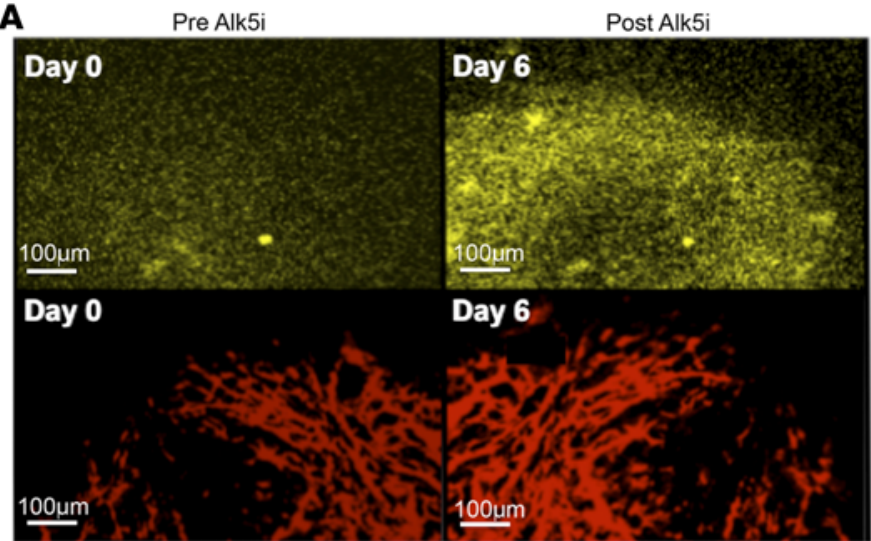

Figure 6. Increased accumulation of indocyanine green in MMTVPyMT tumors following Alk5 inhibition. (A) Representative intravital microscopy images confirm markedly increased indocyanine green (ICG) tumor delivery after Alk5 inhibition (top), while tumor vascularity (bottom) did not change. Scale bar: $100 \mu \mathrm{m}$.

(B) Corresponding quantitative data demonstrate a significantly increased ICG fluorescence signal in Alk5-treated tumors compared with sham-treated controls. Data are displayed as mean tumor fluorescence and SD of 4 animals in each group before and after ICG injection. At least 3 nonoverlapping fields of immunofluorescence images were counted per animal. ANOVA was used to compare the differences between various groups. ${ }^{*} P<0.05$. Results are representative of 3 independent experiments. Alk5, activin-like kinase 5 ; Alk5i, Alk5 inhibition; MMTV-PyMT, mouse mammary tumor virusdriven expression of the polyoma middle T oncogene.

44). TGF- $\beta$ also induces the expression of angiopoietin-like protein 4 (Angpt-4), which, in turn, disrupts endothelial cell junctions, increases leakage, and promotes tumor cell emigration and metastasis to the lungs (45). In addition, it has been shown that ectopic expression of TGF- $\beta 1$ decreases vascular permeability and leakage of Evans blue into the basal keratinocytes of the skin (18). We observed that Alk5 inhibition increases tumor microvascular permeability by about 2.6 -fold and reduces IFP by about $20 \%$. The 3 - to 4 -fold increase in tumor enhancement suggests that there may be additional mechanisms, which will be the focus of further investigations. Other potential mechanisms involved in increased drug delivery could be due to enhanced endocytosis of albumin-bound drugs (46). A different TGF- $\beta$ receptor, TGF- $\beta$ type II receptor (TGF-BRII) has shown to increase albumin endocytosis (46). According to Sounni et al., the Alk5 inhibitor used in this study predominantly inhibits TGF- $\beta 1$ (18). It is important to note that inhibitors of the TGF- $\beta$ type II pathway may have the opposite effect on drug delivery by inhibiting endocytosis of albumin-bound agents. Caveolae are also involved in albumin transcytosis by endothelial cells (47); however, how they respond to TGF- $\beta$ inhibitors remains unknown, and such mechanisms are beyond the scope of this manuscript.

In this study, we investigated mammary tumors and orthotopic brain tumors, both of which rely on active TGF- $\beta 1 /$ Alk5 signaling (24). The investigated transgenic MMTV-PyMT mouse and human glioblastoma models accurately model vascular and therapeutic responses as observed in human breast and brain cancer $(23,24)$. It will be of interest to see if the "angiogenic switch" plays an important role during Alk5 inhibitor-mediated vessel leakiness. In fact, Sounni et al. have shown (18) that the ALK5 signaling pathway was active in chronically leaky angiogenic vessels, such as those found in neoplastic tissues in two different transgenic models of epithelial carcinogenesis $(48,49)$ that include angiogenic blood vessels in the premalignant tissue of K14-HPV16 and MMTV-PyMT transgenic mice. Consistent with these findings, we observed that, in late-stage tumors (day 75), in which angiogenesis rapidly sets in, Alk5 inhibition increased the tumor delivery of ICG by 3.5 -fold as compared with early tumors (day 50 ), in which Alk5 inhibition increased enhanced ICG by 1.2-fold (Figure 4B). Since vascular beds in tumors vary as a function of organ locale, our approach could be expanded to evaluate susceptibility of vasculature in other tumor types, such as pancreas, prostate, lung, skin, and liver cancer.

Since TGF- $\beta 1$ is overexpressed in many malignant tumors but not homeostatic tissues $(33,40,50)$, TGF- $\beta 1$ blockade provides a distinct advantage over other approaches, as it selectively increases contrast agent delivery to tumors. Consistently we observed that Alk5 inhibition caused enrichment of macromolecular drugs selectively in tumors but not normal organs. This increases the tumor-to-background contrast for diagnostic approaches and decreases potential toxic side effects to normal organs for theranostic applications. Importantly, our studies demonstrate a dose-dependent enhancement in contrast agent uptake following Alk5 inhibition with different increasing doses. Future studies will have to optimize dose and 
A

$$
\text { Control (PBS day7) }
$$
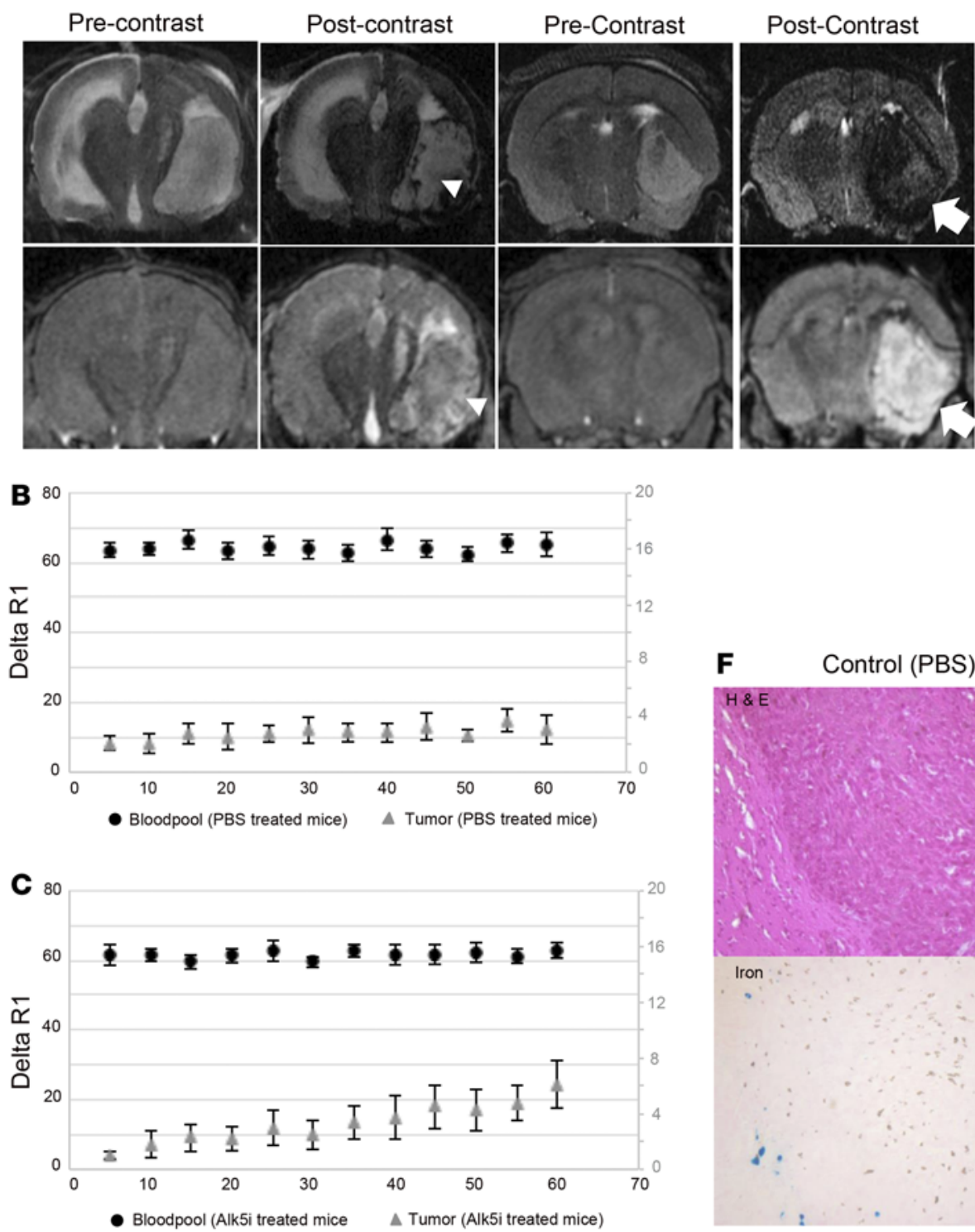

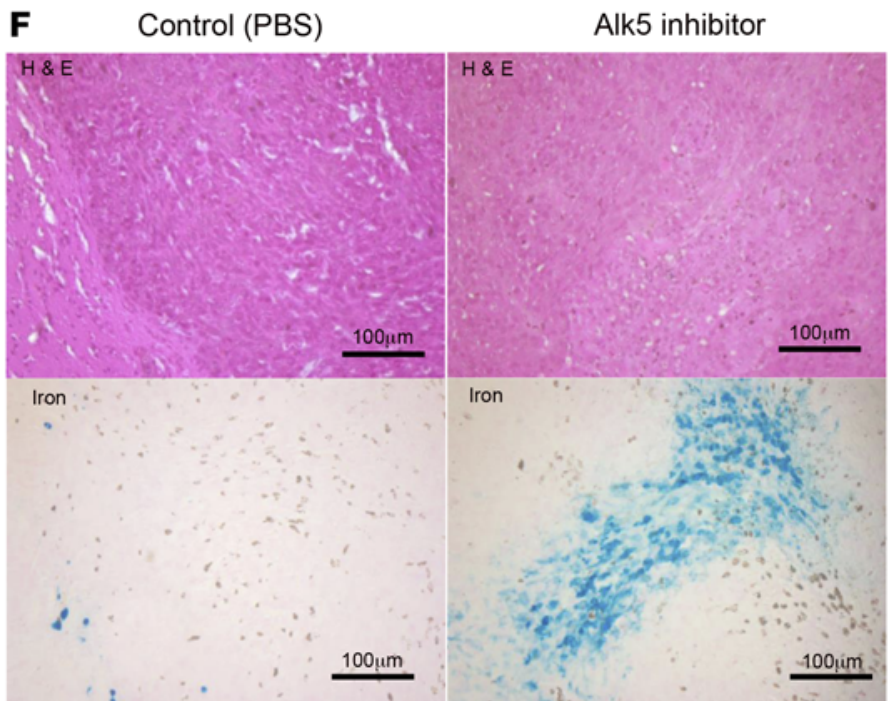

Figure 7. Alk5 inhibition leads to increased iron oxide nanoparticle accumulation and enhancement of orthotopic glioblastomas on MR images. (A) Representative coronal T2-weighted MR images (top) and T1-weighted MR images (bottom) before and after i.v. injection of ferumoxytol nanoparticles show stronger tumor enhancement after Alk5 inhibition (arrow) compared with untreated tumors (arrowhead). (B and C) Representative plots for dynamic contrast ferumoxytol scans in blood pool versus tumor tissue of mice treated with or without Alk5 inhibitor. $\triangle \mathrm{R} 1$ values for blood pool from PBS- or Alk5treated mice are represented on the primary $y$ axis, whereas $\Delta R 1$ values for tumors are represented on secondary $y$ axis. (D and $E$ ) Corresponding quantitative changes in tumor R2 and R1 relaxation rates after ferumoxytol injection, measured as R2 $2_{\text {precontrast }}-\mathrm{R} 2_{\text {postcontrast }}$ or R1 $1_{\text {precontrast }}-\mathrm{R} 1_{\text {postcontrast }}$ and displayed as means for 9 animals in each group $\pm S D$, respectively. ANOVA was used to compare the differences between various groups. ${ }^{*} P<0.05$, ${ }^{* *} P<0.005$, between MR enhancements of Alk5-treated tumors compared with untreated controls. (F) H\&E and Prussian blue staining of a representative Alk5 inhibitor-treated glioblastoma (right) and untreated control (left) mice (original magnification, $\times 20$ ). Prussian blue staining shows increased ferumoxytol deposition in Alk5 inhibitor-treated glioblastoma. Scale bar: $100 \mu \mathrm{m}$. Alk5, activin-like kinase 5; Alk5i, Alk5 inhibition; MR, magnetic resonance

timing of Alk5 inhibition, with regards to imaging studies. A study by van Beuge et al. demonstrated that mice receiving Alk5 inhibitor at $1.3 \mathrm{mg} / \mathrm{kg}$ had reduced levels of TGF- $\beta$-induced genes within 24 hours after Alk5 inhibition (51), whereas Sounni et al., continued the treatment for 7 days at a $1.0 \mathrm{mg} / \mathrm{kg}$ dose to obtain optimum inhibition of the TGF- $\beta /$ Alk5 pathway (18). We noted a variable degree of tumor response to Alk5 inhibitor treatment in terms of tumor contrast enhancement. Therefore, Alk5 inhibition in combination with the predictive power of MRI could identify tumors that benefit from Alk5 inhibi- 
tion to improve outcome. It has been demonstrated that, in mice that have a compromised TGF- $\beta /$ Alk5 pathway, vessel stability is significantly compromised, as evidenced by their increased steady state level of plasma protein leakage (18). Therefore, tumors with a compromised Alk5 pathway would be expected to have significantly increased steady state leakiness, which would make increases in Alk5 inhibition difficult. Detailed evaluations of the underlying mechanisms and implications exceeded the scope of our investigations and have to be addressed in future studies.

Since clinical trials of adjuvant treatments are expensive and require years to complete, our imaging approach offers immediate value and impact for monitoring tumor response to Alk5 inhibitors. Alk5 inhibitors are currently being investigated in clinical trials, for which imaging is the clinical endpoint, for efficacy in suppressing early tumor growth and metastasis (52). Our data have immediate and important clinical implications. There are several ongoing clinical trials that test the safety and efficacy of Alk5 kinase inhibitors on the delivery of therapeutic drugs to malignant tumors (clinicaltrials.gov identifiers NCT02160106, NCT02423343, NCT00656825, NCT02452008, NCT01402193, NCT00356460, NCT02688712, NCT02178358). Imaging scans are typically used to monitor therapy response. A positive response is usually diagnosed based on a decline in tumor contrast enhancement on CT and MRI scans. However, based on our results, a response to Alk5 kinase inhibition would result in an increased contrast agent enhancement of the treated tumor on imaging scans. This information is very important for radiologists to know in order to avoid misdiagnoses of pseudoprogression (imaging findings that look like progression but are not) and related therapy management decisions. We have shown previously that tumor contrast agent enhancement correlated with the delivery of chemotherapeutic agents (2). Therefore, limited tumor contrast enhancement on pretherapeutic tumor staging scans might be used to stratify patients with malignant tumors to a conditioning therapy with Alk5 kinase inhibitors prior to start of their chemotherapy. The efficacy of this conditioning intervention could be monitored with imaging as described here.

Importantly, based on data provided herein, it is reasonable to conclude that the tumor response to Alk5 inhibitors will yield increased tumor contrast enhancement rather than decreased tumor enhancement, typically observed in response to other therapeutic drugs. In summary, our data revealed that Alk5 blockade could be exploited to enhance delivery and accumulation of macromolecular contrast agents of different sizes in malignant tumors. This pathway holds significant promise to improve tumor delivery of tumor-targeted macromolecular contrast agents and nanocarriers and, thereby, may facilitate clinical delivery of more sensitive and specific imaging tests and personalized, image-guided therapies.

\section{Methods}

Eighty transgenic MMTV-PyMT mice bearing late-stage mammary adenocarcinomas and non-tumor-bearing age-matched littermate controls were used (Table 1). Sixteen MMTV-PyMT transgenic mice that spontaneously develop multifocal mammary adenocarcinomas were used for optical imaging (48). In this model of de novo mammary carcinogenesis, the MMTV drives expression of the PyMT, eliciting multistage progression from hyperplasia to invasive carcinoma and metastatic disease in an angiogenic-dependent manner, with $100 \%$ penetrance in female mice within 95 days $(20,53)$. Sixteen age-matched negative littermate control mice served as non-tumor-bearing controls. Additional experiments were carried out in 48 female syngeneic FVB/n mice (10-12 weeks) implanted with explants derived from mammary tumors of MMTV-PyMT mice $(21,22)$. Dose-response studies were conducted with 20 syngeneic FVB/n mice (6-8 weeks) implanted with MMTV-PyMT tumors onto mammary fat pad. To prove the concept of increased contrast agent delivery after Alk5 inhibition, human glioblastoma cells D270 were harvested from human brain tumor samples, enriched for cancer stem cells, and stereotactically implanted into the left brain hemisphere of $36 \mathrm{NOD} / \mathrm{SCID} \times \mathrm{RAG} \gamma / \delta$ double-knockout mice. The D270 glioblastoma cells were a gift from Samuel Cheshier, Stanford University, Stanford, California, USA.

Mice were randomized to experimental groups that underwent intravital microscopy, MRI, or IFP measurements, as detailed in Table 1 and ref. 54. Pharmacological inhibition of Alk5 was achieved using the Alk5 kinase inhibitor [3-(pyridin-2-yl)-4-(4-quinonyl)]-1H-pyrazole (Calbiochem) administered i.p. at $1.0 \mathrm{mg} / \mathrm{kg}$ in $2 \%$ DMSO in sterile PBS every other day for 6 days prior to imaging and necropsy (18). Control mice received equivalent i.p. injections of PBS alone. Animal age, weight, and tumor size did not significantly vary between experimental groups $(P>0.05)$.

MRI before and after Alk5 inhibition. Alk5 inhibitor-treated or untreated MMTV-PyMT tumor-bearing 
mice underwent serial MRI studies before and after i.v. injection of 2 different, clinically applicable, macromolecular MR contrast agents:

We first evaluated gadofosveset trisodium $\left(\mathrm{C}_{33} \mathrm{H}_{40} \mathrm{GdN}_{3} \mathrm{Na}_{3} \mathrm{O}_{15} \mathrm{P}\right.$; Ablavar, Lantheus Medical Imaging, dose $0.05 \mathrm{mmol} \mathrm{Gd} / \mathrm{kg}$ ), an FDA-approved contrast agent for MRI (25). Gadofosveset trisodium has a molecular weight of $975.88 \mathrm{~g} / \mathrm{mol}$ and binds $>90 \%$ to plasma albumin, thereby forming macromolecules of approximately 80 to $90 \mathrm{kDa}$ in vivo. The nonprotein bound moiety is excreted via the kidneys with a blood half life of approximately 20 minutes, thereby allowing follow-up imaging studies within a short time interval. Thus, 6 tumor-bearing mice underwent gadofosveset-enhanced MRI before (day 1) and after (day 7) Alk5 inhibitor treatment. Six additional tumor-bearing control mice underwent gadofosveset-enhanced MRI before (day 1) and after (day 7) treatment with PBS. Gadofosveset-enhanced MRI was obtained directly before and after gadofosveset injection. Experiments were done in triplicate.

We next evaluated tumor delivery of the nanoparticle-based FDA-approved iron supplement ferumoxytol (AMAG Pharmaceuticals; dose $0.5 \mathrm{mmol} \mathrm{Fe} / \mathrm{kg}$ ) (26) in MMTV-PyMT mice. Ferumoxytol is composed of iron oxide nanoparticles with a mean hydrodynamic diameter of $30 \mathrm{~nm}$ that provide superparamagnetic signal effects on T1- and T2-weighted MR images; ferumoxytol, therefore, can be used "off label" as a clinical MR contrast agent $(55,56)$. Ferumoxytol nanoparticles have a blood half life of several hours and are retained in tumor tissue and the reticuloendothelial system, where they are slowly metabolized by macrophages (21). Due to this slow clearance, imaging studies before and after Alk5 treatment were performed in different animals; MMTV-PyMT tumor-bearing mice before $(n=4)$ and after $(n=4)$ Alk5 inhibitor treatment as well as before $(n=4)$ and after $(n=4)$ PBS treatment underwent MRI before and at 1 hour and 24 hours after ferumoxytol injection. Two additional mice, which received Alk5 inhibitor or PBS but no contrast agent served as controls in each imaging study. Similarly glioblastoma-bearing mice before and after Alk5 inhibitor $(n=9)$ or PBS $(n=9)$ treatment underwent MRI before and at 1 hour and 24 hours after ferumoxytol injection. Experiments were done in triplicate.

MRI studies of MMTV-PyMT mice were performed on a 1T desktop MR scanner (Aspect M2Ô Compact High Performance MR System), using T1- and T2-weighted imaging sequences with the following parameters: T1 inversion recovery - TR 2,000 ms, TE $12 \mathrm{~ms}$, TI 20-500 ms; and T2 spin echo - TR 2,500 $\mathrm{ms}$, TE 20,40,60, $80 \mathrm{~ms}$. MR scans were obtained with a field of view of $6 \times 6 \mathrm{~cm}$, a matrix of $128 \times 128$ or $200 \times 200$ pixels, and a slice thickness of 1 to $2 \mathrm{~mm}$.

MRI studies of glioblastoma-bearing mice were performed on a 7T MR scanner (Agilent Technologies-General Electric Healthcare), using T1- and T2-weighted imaging sequences, with a field of view of 2 $\mathrm{cm} \times 2 \mathrm{~cm}$ and a slice thickness of 0.3 to $1 \mathrm{~mm}$, with the following parameters: T2 FSE (TR: 6,000 ms, TE: $90 \mathrm{~ms}, \alpha: 90^{\circ}$ ), T2 FGRE (TR: $300 \mathrm{~ms}$, TE: 2.4, 3.9, 4.6, 5.4, 6.1, 7.6 ms, $\alpha: 30^{\circ}$ ), and T1 3D FSPGR (TR: $7.8 \mathrm{~ms}$, TE: $1.8 \mathrm{~ms}, \alpha: 5^{\circ}$ ).

$\mathrm{T} 1$ and $\mathrm{T} 2$ relaxation times of the tumors as well as liver and muscle as internal standards were calculated and converted to $\mathrm{R} 1$ and $\mathrm{R} 2$ relaxation rates $(\mathrm{R} 2=1 / \mathrm{T} 2)$, which are proportional to contrast agent concentrations. The relative change in relaxation rates between MR scans before and after contrast, $\Delta \mathrm{R} 1$ and $\Delta \mathrm{R} 2(\%)$, were determined as a quantitative measure of tumor contrast enhancement.

Histopathologic correlations of MRI data. For Prussian blue iron stains, tissue sections (5 $\mu \mathrm{m}$ ) of formalin-fixed, paraffin-embedded tissue were deparaffinized with xylene, rehydrated, and stained according to the manufacturer's recommendation with the Sigma-Aldrich Accustain Iron Stain Kit. Signal was amplified with Fast 3,3 diaminobenzidine (DAB, Vector Laboratories) for 2 minutes, and sections were counterstained with Methyl Green (Fisher Scientific). Representative images were captured using the Aperio ScanScope CS Slide Scanner with a $\times 40$ objective for whole-slide imaging. For immunofluorescence, formalin-fixed, paraffin-embedded sections were deparaffinized with xylene and rehydrated and peroxidase activity was blocked with methanol $/ 0.006 \% \mathrm{H}_{2} \mathrm{O}_{2}$ for 20 minutes. After washing 3 times in PBS, sections were immersed in proteinase K solution (Dako) for 3 minutes. Sections were washed 3 times with PBS and blocked for 1 hour with PBS containing 5\% normal goat serum and 2.5\% BSA. Sections were immunofluorescently stained with biotinylated CD31 antibody clone MEC13.3 (1:1,000 dilution, Biolegend), Alk5, and phospho-Smad2 antibody 3101 (1:500 dilution, Cell Signaling) overnight at $4^{\circ} \mathrm{C}$. CD31 was amplified using tyramide signal amplification using the Perkin-Elmer TSA Indirect Kit according to the manufacturer's recommendation. Signal was detected with streptavidin-Alexa Fluor 488 and goat anti-rabbit-Alexa Fluor 555 (Life Technologies) and counterstained with Hoechst 33342. Slides were coverslipped with Vectastain mounting medium (Vector Laboratories). Images were captured using a Zeiss Apotome fluorescent 
microscope and Zen 2011 software using the same exposure, gain, and threshold parameters to allow direct comparisons of staining patterns. Velocity software was further used to quantify the degree of Alk5 inhibition. The number of cells with maximum Alk5 expression was calculated per unit area for control and Alk5 inhibitor-treated groups and compared using 2-tailed Student's $t$ test. Mean signal intensity for each treatment group was also calculated. Sections from 3 different animals per treatment group (mean \pm SD) were used.

Tumor contrast enhancement on optical images before and after Alk5 inhibition. ICG is an FDA-approved, hydrophilic, anionic, tricarbocyanine dye with a molecular weight of $774.97 \mathrm{Da}$ and strong fluorescence in the near-infrared spectrum (ex/em: 780/830 nm; ref. 57). ICG binds $>90 \%$ to plasma albumin, thereby representing a macromolecule in vivo. The agent is eliminated via hepatobiliary excretion. Sixteen additional transgenic MMTV-PyMT mice with early-stage (day 50, $n=8$ ) or late-stage mammary adenocarcinomas (day 75,n=8) underwent optical imaging before and after treatment with Alk5 inhibitor ( $n=4$ per group). Sixteen non-tumor-bearing mice served as controls. Optical imaging was performed before and at various time points after i.v. injection of ICG (Sigma-Aldrich, dose: $7 \mathrm{mg} / \mathrm{kg}$ ). This dose was chosen based on a study by Meier et al., which showed that high ICG doses lead to prolonged tissue enhancement on optical images (58). We also performed a dose-response study in which 20 mice bearing MMTV-PyMT adenocarcinomas received PBS $(n=4)$ or Alk5 inhibitor ( $n=4 ; 0.5,1,2$, and $4 \mathrm{mg} / \mathrm{kg}$ body weight) every other day for 6 days prior to imaging with ICG.

Intravital microscopy of ICG tumor delivery before and after Alk5 inhibition. Eight additional FVB/n mice were implanted with one million MMTV-PyMT tumor-derived cells into dorsal window chambers and underwent intravital microscopy before and after Alk5 inhibition. When animals developed tumors with a size of 5 to $7 \mathrm{~mm}$, they were anesthetized with isoflurane, and tumors were positioned and stabilized beneath the objective on an IV-100 intravital microscope (Olympus IV100). Long-circulating Angiosense 750 dye (PerkinElmer, molecular weight $250 \mathrm{kDa}$, dose $2 \mathrm{mg} / \mathrm{kg}$, ex/em 750/780) was injected via the tail vein to outline vasculature, followed by i.v. injections of ICG (dose $3.0 \mathrm{mg} / \mathrm{kg}$, ex/em 805/830). Mice were imaged during injection and until ICG was no longer visible in the vasculature. Output channels for Angiosense and ICG were scanned sequentially to prevent filter bleed-through. Extravasation was analyzed by reference to the presence of ICG signal outside Angiosense-marked blood vessels, using a region-of-interest (ROI) analysis. Z-stacks were generated by optically sectioning through the ROI (typically 10-20 sections per region) at every time point. For each mouse, at early and late time points in the same field of view, 4 to 8 ROIs were placed outside of blood vessels. Data from the 4 to 8 ROIs were averaged for each mouse, and the resultant average was averaged for all mice in the group and computed with standard errors of the mean. Image J software was used for quantification of vessel density. Vessel quantification was expressed as vessel density $\left(\mathrm{mm}^{2}\right)$. Sections from 3 different animals per treatment group (mean $\pm \mathrm{SD}$ ) were used.

Measurement of tumor interstitial pressure before and after Alk5 inhibition. Sixteen additional MMTV-PyMT mammary tumor-bearing mice, before and after Alk5 inhibitor treatment $(n=8)$ or PBS treatment $(n=8)$, underwent in vivo measurements of tumor IFP via the "wick-in-needle technique" $(27,59)$. This procedure uses a fine hollow steel needle filled with isotonic saline connected to an APT300 Pressure Transducer and a TAM-D Transducer amplifier (Harvard Apparatus). The transducer dome has a second connection for flushing the needle (closed by an arterial clamp during measurement). Tumor IFP measurements were related to tumor volume, which was measured by a caliper in 3 dimensions. Mice were anesthetized with isofluorane and placed on a regulated heating pad to maintain body temperature at $38^{\circ} \mathrm{C}$, and the tumor was punctured using the hollow needle (15 gauge). Fluid communication between the needle and tumor tissue was confirmed by compressing and decompressing tubing, and data were accepted only when recorded pressures returned rapidly to the precompression values. The tumor IFP was measured at least 3 times for each tumor, normalized to control measurements in the subcutis $(0-1 \mathrm{~mm} \mathrm{Hg})$, and averaged for tumors before and after Alk5 inhibitor treatment.

Statistics. Tumor and organ relaxation rates and fluorescence signals were compared within multiple experimental groups using ANOVA. A 2-tailed Student's $t$ test was used to compare differences in age, tumor size, and tumor IFP of mice in different experimental groups. A $P$ value of less than 0.05 was considered significant.

Study approval. The study was approved by the animal care and use committees at UCSF and Stanford University (Palo Alto, California, USA) and was performed in accordance with the NIH Guide for the Care and Use of Laboratory Animals. 


\section{Author contributions}

HEDL, LMC, and CA designed the experiments. CA, SM, OL, AS, KI, SHH, MH, and LP performed the experiments. CA, OL, and SM analyzed the data. NB, SSG, and LMC reviewed the data and edited the manuscript. HEDL, CA, and SM wrote the manuscript.

\section{Acknowledgments}

The authors thank the Small Animal Imaging Facility at Stanford for providing the equipment and infrastructure for this project. We thank members of the Daldrup-Link, Gambhir, and Coussens laboratories for valuable discussions. We acknowledge support from NIH National Cancer Institute (NCI) grant R01 CA140943 (to L.M. Coussens, H.E. Daldrup-Link, and N. Boudreau); grants R21 CA176519 (to H.E. Daldrup-Link), R21 CA190196-01A1, R01 CA155331, and U54 CA163123 from the NCI; the Department of Defense Breast Cancer Research Program Era of Hope Scholar Expansion Award (BC10412); the Susan G. Komen Foundation; Stand Up To Cancer - Lustgarten Foundation Pancreatic Cancer Convergence Dream Team Translational Research Grant; and the Breast Cancer Research Foundation (to L.M. Coussens).

Address correspondence to: Heike E. Daldrup-Link, 725 Welch Rd., Rm 1665, Stanford, California $94305-$ 5614, USA. Phone: 650.723.8996; E-mail: H.E.Daldrup-Link@stanford.edu.

1. Albanese A, Tang PS, Chan WC. The effect of nanoparticle size, shape, and surface chemistry on biological systems. Annu Rev Biomed Eng. 2012;14:1-16.

2. Daldrup-Link HE, et al. Decrease in tumor apparent permeability-surface area product to a MRI macromolecular contrast medium following angiogenesis inhibition with correlations to cytotoxic drug accumulation. Microcirculation. 2004;11(5):387396.

3. Daldrup-Link HE, Simon GH, Brasch RC. Imaging of tumor angiogenesis: current approaches and future prospects. Curr Pharm Des. 2006;12(21):2661-2672.

4. Dreaden EC, Mackey MA, Huang X, Kang B, El-Sayed MA. Beating cancer in multiple ways using nanogold. Chem Soc Rev. 2011;40(7):3391-3404

5. Ferrara K, Pollard R, Borden M. Ultrasound microbubble contrast agents: fundamentals and application to gene and drug delivery. Annu Rev Biomed Eng. 2007;9:415-447.

6. Hellebust A, Richards-Kortum R. Advances in molecular imaging: targeted optical contrast agents for cancer diagnostics. Nanomedicine (Lond). 2012;7(3):429-445.

7. Janib SM, Moses AS, MacKay JA. Imaging and drug delivery using theranostic nanoparticles. Adv Drug Deliv Rev. 2010;62(11):1052-1063.

8. Workman P, et al. Minimally invasive pharmacokinetic and pharmacodynamic technologies in hypothesis-testing clinical trials of innovative therapies. J Natl Cancer Inst. 2006;98(9):580-598.

9. Zhou Z, Lu ZR. Gadolinium-based contrast agents for magnetic resonance cancer imaging. Wiley Interdiscip Rev Nanomed Nanobiotechnol. 2013;5(1):1-18.

10. Dreher MR, Liu W, Michelich CR, Dewhirst MW, Yuan F, Chilkoti A. Tumor vascular permeability, accumulation, and penetration of macromolecular drug carriers. J Natl Cancer Inst. 2006;98(5):335-344.

11. Jain RK. Barriers to drug delivery in solid tumors. Sci Am. 1994;271(1):58-65.

12. Minchinton AI, Tannock IF. Drug penetration in solid tumours. Nat Rev Cancer. 2006;6(8):583-592.

13. Netti PA, et al. Enhancement of fluid filtration across tumor vessels: implication for delivery of macromolecules. Proc Natl Acad Sci U S A. 1999;96(6):3137-3142.

14. Torchilin V. Tumor delivery of macromolecular drugs based on the EPR effect. Adv Drug Deliv Rev. 2011;63(3):131-135.

15. Dvorak HF. Vascular permeability factor/vascular endothelial growth factor: a critical cytokine in tumor angiogenesis and a potential target for diagnosis and therapy. J Clin Oncol. 2002;20(21):4368-4380.

16. Lejeune FJ. Clinical use of TNF revisited: improving penetration of anti-cancer agents by increasing vascular permeability. $J$ Clin Invest. 2002;110(4):433-435.

17. Muldoon LL, Gahramanov S, Li X, Marshall DJ, Kraemer DF, Neuwelt EA. Dynamic magnetic resonance imaging assessment of vascular targeting agent effects in rat intracerebral tumor models. Neuro Oncol. 2011;13(1):51-60.

18. Sounni NE, et al. Stromal regulation of vessel stability by MMP14 and TGF $\beta$. Dis Model Mech. 2010;3(5-6):317-332.

19. Akhurst RJ, Hata A. Targeting the TGF $\beta$ signalling pathway in disease. Nat Rev Drug Discov. 2012;11(10):790-811.

20. Lin EY, et al. Macrophages regulate the angiogenic switch in a mouse model of breast cancer. Cancer Res. 2006;66(23):1123811246.

21. Daldrup-Link HE, et al. MRI of tumor-associated macrophages with clinically applicable iron oxide nanoparticles. Clin Cancer Res. 2011;17(17):5695-5704.

22. DeNardo DG, et al. Leukocyte complexity in breast cancer predicts overall survival and functionally regulates response to chemotherapy. Cancer Discov. 2011;1(1):54-67.

23. Lin EY, et al. Progression to malignancy in the polyoma middle T oncoprotein mouse breast cancer model provides a reliable model for human diseases. Am J Pathol. 2003;163(5):2113-2126.

24. Joseph JV, Balasubramaniyan V, Walenkamp A, Kruyt FA. TGF- $\beta$ as a therapeutic target in high grade gliomas - promises and challenges. Biochem Pharmacol. 2013;85(4):478-485. 
25. Haneder S, et al. Gadofosveset: parameter optimization for steady-state imaging of the thoracic and abdominal vasculature. Invest Radiol. 2011;46(11):678-685.

26. Lu M, Cohen MH, Rieves D, Pazdur R. FDA report: Ferumoxytol for intravenous iron therapy in adult patients with chronic kidney disease. Am J Hematol. 2010;85(5):315-319.

27. Hofmann M, et al. Lowering of tumor interstitial fluid pressure reduces tumor cell proliferation in a xenograft tumor model. Neoplasia. 2006;8(2):89-95.

28. Smith BR, et al. Shape matters: intravital microscopy reveals surprising geometrical dependence for nanoparticles in tumor models of extravasation. Nano Lett. 2012;12(7):3369-3377.

29. Goel S, et al. Normalization of the vasculature for treatment of cancer and other diseases. Physiol Rev. 2011;91(3):1071-1121.

30. Jain RK. Normalization of tumor vasculature: an emerging concept in antiangiogenic therapy. Science. 2005;307(5706):58-62.

31. Carmeliet P, Jain RK. Principles and mechanisms of vessel normalization for cancer and other angiogenic diseases. Nat Rev Drug Discov. 2011;10(6):417-427.

32. Bergers G, Hanahan D. Modes of resistance to anti-angiogenic therapy. Nat Rev Cancer. 2008;8(8):592-603.

33. Chauhan VP, et al. Normalization of tumour blood vessels improves the delivery of nanomedicines in a size-dependent manner Nat Nanotechnol. 2012;7(6):383-388.

34. Cole AJ, Yang VC, David AE. Cancer theranostics: the rise of targeted magnetic nanoparticles. Trends Biotechnol. 2011;29(7):323-332

35. Corot C, Robert P, Idee JM, Port M. Recent advances in iron oxide nanocrystal technology for medical imaging. Adv Drug Deliv Rev. 2006;58(14):1471-1504.

36. Daldrup-Link HE, Brasch RC. Macromolecular contrast agents for MR mammography: current status. Eur Radiol. 2003;13(2):354-365

37. Bhattacharya $\mathrm{D}$, et al. Small molecule-directed immunotherapy against recurrent infection by Mycobacterium tuberculosis. $J$ Biol Chem. 2014;289(23):16508-16515.

38. Salnikov AV, Roswall P, Sundberg C, Gardner H, Heldin NE, Rubin K. Inhibition of TGF-beta modulates macrophages and vessel maturation in parallel to a lowering of interstitial fluid pressure in experimental carcinoma. Lab Invest. 2005;85(4):512521.

39. Kano MR, et al. Improvement of cancer-targeting therapy, using nanocarriers for intractable solid tumors by inhibition of TGF- $\beta$ signaling. Proc Natl Acad Sci U S A. 2007;104(9):3460-3465

40. Liu J, et al. TGF- $\beta$ blockade improves the distribution and efficacy of therapeutics in breast carcinoma by normalizing the tumor stroma. Proc Natl Acad Sci U S A. 2012;109(41):16618-16623.

41. Madri JA, Bell L, Merwin JR. Modulation of vascular cell behavior by transforming growth factors $\beta$. Mol Reprod Dev. 1992;32(2):121-126

42. Cortes-Funes H. The role of antiangiogenesis therapy: bevacizumab and beyond. Clin Transl Oncol. 2009;11(6):349-355

43. Pittet JF, et al. TGF- $\beta$ is a critical mediator of acute lung injury. J Clin Invest. 2001;107(12):1537-1544

44. Su G, et al. Integrin $\alpha v \beta 5$ regulates lung vascular permeability and pulmonary endothelial barrier function. Am J Respir Cell Mol Biol. 2007;36(3):377-386.

45. Padua D, et al. TGF $\beta$ primes breast tumors for lung metastasis seeding through angiopoietin-like 4. Cell. 2008;133(1):66-77.

46. Siddiqui SS, Siddiqui ZK, Malik AB. Albumin endocytosis in endothelial cells induces TGF- $\beta$ receptor II signaling. Am J Physiol Lung Cell Mol Physiol. 2004;286(5):L1016-L1026.

47. $\mathrm{Li} \mathrm{HH}$, et al. Caveolae-dependent and -independent uptake of albumin in cultured rodent pulmonary endothelial cells. PLoS One. 2013;8(11):e81903

48. Guy CT, Cardiff RD, Muller WJ. Induction of mammary tumors by expression of polyomavirus middle T oncogene: a transgenic mouse model for metastatic disease. Mol Cell Biol. 1992;12(3):954-961.

49. Coussens LM, Hanahan D, Arbeit JM. Genetic predisposition and parameters of malignant progression in K14-HPV16 transgenic mice. Am J Pathol. 1996;149(6):1899-1917.

50. Blobe GC, Schiemann WP, Lodish HF. Role of transforming growth factor beta in human disease. $N$ Engl J Med. 2000;342(18):1350-1358.

51. van Beuge MM, et al. Enhanced effectivity of an ALK5-inhibitor after cell-specific delivery to hepatic stellate cells in mice with liver injury. PLoS One. 2013;8(2):e56442.

52. Bhola NE, et al. TGF- $\beta$ inhibition enhances chemotherapy action against triple-negative breast cancer. J Clin Invest. 2013;123(3):1348-1358.

53. Crowther M, Brown NJ, Bishop ET, Lewis CE. Microenvironmental influence on macrophage regulation of angiogenesis in wounds and malignant tumors. J Leukoc Biol. 2001;70(4):478-490.

54. Varticovski L, et al. Accelerated preclinical testing using transplanted tumors from genetically engineered mouse breast cancer models. Clin Cancer Res. 2007;13(7):2168-2177.

55. Neuwelt EA, et al. The potential of ferumoxytol nanoparticle magnetic resonance imaging, perfusion, and angiography in central nervous system malignancy: a pilot study. Neurosurgery. 2007;60(4):601-611.

56. Simon GH, et al. Ultrasmall supraparamagnetic iron oxide-enhanced magnetic resonance imaging of antigen-induced arthritis: a comparative study between SHU 555 C, ferumoxtran-10, and ferumoxytol. Invest Radiol. 2006;41(1):45-51.

57. Shi C, Zhang C, Su Y, Cheng T. Cyanine dyes in optical imaging of tumours. Lancet Oncol. 2010;11(9):815-816.

58. Meier R, et al. Detection of postoperative granulation tissue with an ICG-enhanced integrated OI-/X-ray System. J Transl Med. 2008;6:73.

59. Hofmann M, et al. Increased plasma colloid osmotic pressure facilitates the uptake of therapeutic macromolecules in a xenograft tumor model. Neoplasia. 2009;11(8):812-822. 